\title{
Práticas integrativas e complementares na atenção básica: Revisão integrativa da
}

\section{literatura}

\author{
Integrative and complementary practices in primary care: Integrative literature review \\ Prácticas integradoras y complementarias en atención primaria: Revisión integrativa de la
}

literatura

Recebido: 13/07/2021 | Revisado: 19/07/2021 | Aceito: 22/07/2021 | Publicado: 29/07/2021

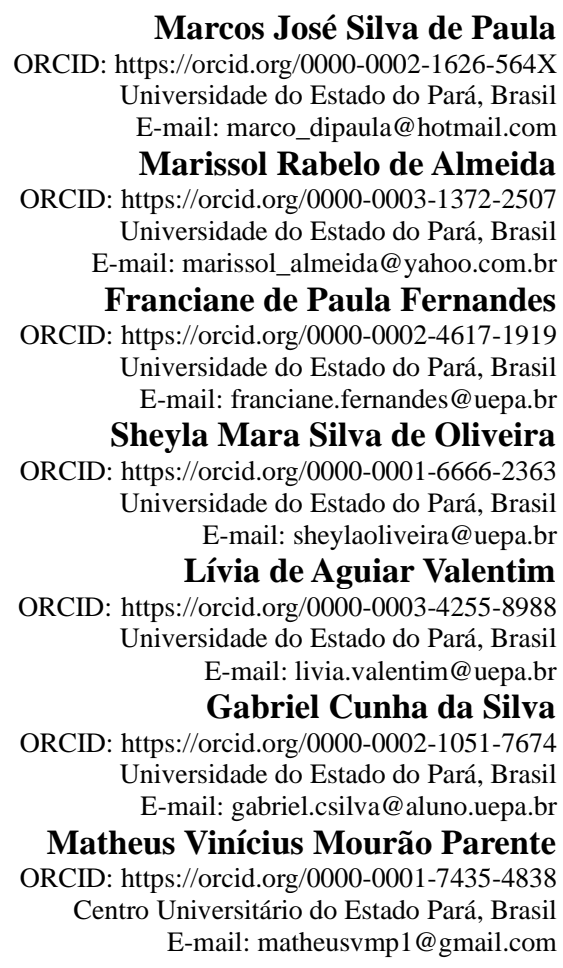

\section{Resumo}

As práticas integrativas e complementares buscam incentivar a utilização de maneiras naturais de prevenção de doenças, promoção, manutenção e recuperação da saúde, por meio de práticas seguras e eficazes. Esses sistemas enfatizam o modo acolhedor de escuta, a formação de vínculo terapeuta-paciente e a atenção na integral da pessoa com a sociedade e o meio ambiente. O objetivo geral deste estudo foi analisar o cenário de produção científica sobre práticas integrativas e complementares com ênfase na atenção primária à saúde. Trata-se de revisão da literatura, do tipo exploratória nas seguintes bases de dados: Scientific Eletronic Library Online (SciELO) e Biblioteca Virtual em saúde (BVS). A coleta dos dados ocorreu de janeiro a março de 2019. Foram encontrados 148 estudos científicos, sendo 101 na base de dados BVS e 47 na SciELO. Após a leitura, foram selecionados aqueles que atendiam os objetivos da pesquisa. Verificou-se que o maior número de publicações de acordo com a região geográfica é proveniente das regiões sudeste, nordeste e sul. Sobre temas mais abordados nos estudos, emergiram 3 categorias temáticas: processo de implantação das PICS na atenção básica, aplicação das PICs para variadas finalidades no contexto da atenção básica e formação/capacitação dos profissionais da saúde envolvidos. Concluiu-se, por meio dos estudos aqui considerados, um cenário em que as PICs estão em ascensão cada vez mais sendo objeto de pesquisas, e isso nos apresenta à relevância da implementação desse modelo de serviços na atenção básica.

Palavras-chave: Terapias complementares; Atenção primária à saúde; Saúde pública.

\section{Abstract}

Integrative and complementary practices seek to encourage the use of natural ways to prevent diseases, promote, maintain and recover health, through safe and effective practices. These systems emphasize the welcoming way of listening, the formation of a therapist-patient bond and the person's integral attention to society and the environment. The general objective of this study was to analyze the scenario of scientific production on integrative and 
complementary practices with an emphasis on primary health care. This is an exploratory literature review in the following databases: Scientific Electronic Library Online (SciELO) and Virtual Health Library (VHL). Data collection took place from January to March 2019. 148 scientific studies were found, 101 in the BVS database and 47 in SciELO. After reading, those who met the research objectives were selected. It was found that the largest number of publications according to geographic region comes from the southeast, northeast and south. Three thematic categories emerged on topics covered in the studies: PICS implementation process in primary care, application of PICs for various purposes in the context of primary care and training/training of health professionals involved. It was concluded, through the studies considered here, a scenario in which PICs are increasingly being the object of research, and this introduces us to the relevance of implementing this model of services in primary care.

Keywords: Complementary therapies; Primary health care; Public health.

\section{Resumen}

Las prácticas integradoras y complementarias buscan incentivar el uso de formas naturales para prevenir enfermedades, promover, mantener y recuperar la salud, a través de prácticas seguras y efectivas. Estos sistemas enfatizan la forma acogedora de escuchar, la formación de un vínculo terapeuta-paciente y la atención integral de la persona a la sociedad y el medio ambiente. El objetivo general de este estudio fue analizar el escenario de producción científica sobre prácticas integradoras y complementarias con énfasis en la atención primaria de salud. Se trata de una revisión exploratoria de la literatura en las siguientes bases de datos: Scientific Electronic Library Online (SciELO) y Virtual Health Library (BVS). La recolección de datos se realizó de enero a marzo de 2019. Se encontraron 148 estudios científicos, 101 en la base de datos BVS y 47 en SciELO. Después de la lectura, se seleccionaron aquellos que cumplieron con los objetivos de la investigación. Se encontró que el mayor número de publicaciones según región geográfica proviene del sureste, noreste y sur. Surgieron tres categorías temáticas sobre los temas tratados en los estudios: proceso de implementación del PICS en atención primaria, aplicación de los PIC para diversos fines en el contexto de la atención primaria y formación / formación de los profesionales de la salud involucrados. Se concluyó, a través de los estudios aquí considerados, un escenario en el que los PIC son cada vez más objeto de investigación, y esto nos introduce en la relevancia de implementar este modelo de servicios en atención primaria.

Palabras clave: Terapias complementarias; Primeros auxilios; Salud pública.

\section{Introdução}

Segundo o Departamento de Atenção Básica do Ministério da Saúde, as Práticas Integrativas e Complementares (PICs) estão cada vez mais presentes no Sistema Único de Saúde (SUS), sendo distribuídas pelos 27 estados e pelo Distrito Federal. Atualmente, $78 \%$ da oferta de PICs ocorre na Atenção Básica, com 2 milhões de atendimentos já realizados em Unidades Básicas de Saúde (UBSs), 18\% na atenção secundária e apenas 4\% na atenção terciária (Ministério da saúde, s.d.). Embora as PICS possam ser ofertadas em todos os níveis de atenção à saúde no SUS, a Política Nacional de Práticas Integrativas e Complementares (PNPIC) estimula que essas práticas sejam implantadas prioritariamente na Atenção Básica (Brasil, 2018), que constitui a porta de entrada do usuário e busca promover uma atenção integral ao indivíduo (Brasil, 2011).

As modalidades terapêuticas não-convencionais, as quais abrangem as PICs, têm conquistado cada vez mais espaço em diversos países (Brant et al., 2014). No Brasil, essas terapias, começaram a suscitar debates no final de década de 1970 e principalmente nos anos 1980 com a $8^{\text {a }}$ Conferência Nacional de Saúde, espaço este que deu visibilidade às demandas da população por uma cultura de saúde questionadora do modelo hegemônico de cuidado terapêutico (Ministério da saúde, s.d.).

Os maiores desafios apontados por Habimorad (2015), referem-se à necessidade de formação e qualificação de uma quantidade adequada de profissionais para atuarem no SUS, ações efetivas de monitoramento e avaliação dos serviços de PICs, conforme preconizam as diretrizes gerais da política, além da estruturação dos serviços nos locais de atendimento. Para isso, segundo esse autor, é fundamental a participação política nos fóruns decisórios do SUS e divulgação da PNPIC para maior conhecimento dos gestores e profissionais da rede pública de saúde.

Tendo em vista a relevância das PICs no âmbito do SUS no cenário atual, e que apesar disso, um dos desafios da PNPIC é a difusão do conhecimento junto aos atores envolvidos (gestores, profissionais e usuários da saúde), faz-se necessário estudos que abordem essa temática com o intuito de fortalecer o corpo de conhecimento existente e contribuir para a divulgação dessa política. Assim, determinou-se o seguinte problema de pesquisa: Qual o cenário da produção científica sobre práticas integrativas e complementares na atenção básica evidenciado na literatura nos últimos cinco anos? 
Dessa forma, este estudo tem por objetivo realizar revisão integrativa da literatura acerca das práticas integrativas e complementares no SUS, com enfoque na atenção básica à saúde.

\section{Metodologia}

Segundo Souza, Silva e Carvalho (2010), a revisão integrativa da literatura proporciona uma síntese de conhecimento e a incorporação da aplicabilidade de resultados de estudos significativos na prática.

De acordo com Rodrigues (2006), esta pesquisa pode ser caracterizada: quanto à natureza da pesquisa, é aplicada, pois gerará conhecimentos dirigidos à solução de problemas específicos; quanto à modalidade, é bibliográfica, pois visa à recuperação do conhecimento científico acumulado sobre um determinado tema/problema.; quanto aos objetivos, é exploratória, já que pretende proporcionar mais familiaridade com o problema; quanto à abordagem, é qualitativa, pois se trata de uma pesquisa documental e bibliográfica.

O levantamento desses dados ocorreu entre os meses de janeiro e março de 2019. Foi realizada busca nas bases de dados Biblioteca Virtual em Saúde (BVS) e biblioteca eletrônica Scientific Eletronic Library Online (SciELO). Para a busca dos artigos na BVS foram utilizadas as seguintes combinações de termos: "atenção básica" e o descritor "terapias complementares, assim como "atenção primária" e "terapias integrativas e complementares". Na busca na biblioteca SciELO, foram utilizadas as seguintes combinações de termos: "práticas integrativas e complementares e "atenção básica"; "práticas integrativas e complementares e "atenção primária"; "PIC" e "atenção primária"; além de "práticas integrativas e complementares", "estratégia de saúde da família" e "atenção primária à saúde"

Foram considerados os seguintes critérios de inclusão: textos completos, com acesso nas bases de dados, nos idiomas português, inglês ou espanhol, publicados nos anos de 2014 a 2018 e que abordem temáticas relevantes à pesquisa. Optou-se por esta faixa temporal pelo interesse em identificar quais as discussões mais recentes acerca do tema. Não foram incluídos editoriais, teses, dissertações ou estudos que não abordavam temática relevante ao alcance do objetivo da revisão.

Para a coleta de dados, foi utilizado um formulário para pesquisa documental adaptado de Ursi (2005) (Apêndice A), o qual permitiu uma avaliação mais detalhada e organizada de cada artigo, tanto na metodologia quanto nos resultados destes, sintetizando-os em uma planilha do programa Microsoft Excel @ para uma melhor análise. Esse instrumento contém variáveis divididas em dois eixos: no primeiro consta título do artigo, título do periódico, base de dados, ano, número de autores, idioma, modalidade, tipo de publicação, abordagem, tipo de estudo, número de sujeitos, técnicas de coleta de dados e técnica de análise; já o segundo eixo está voltado para os resultados evidenciados.

Para o preenchimento da planilha, foi realizada a leitura superficial dos títulos, resumos, metodologia resultados e conclusões. Assim, os textos encontrados foram avaliados quanto à adequação a temática proposta. Aqueles que tratavam de outras temáticas foram automaticamente excluídos nesta etapa.

A partir da transcrição dos artigos selecionados na planilha, foi realizada a análise dos dados, com o cálculo de frequência simples, a fim de descrever a caracterização dos artigos encontrados, considerando idioma, ano de publicação, tipo de estudo, local de publicação e tipo de abordagem.

Posteriormente, no que tange à segunda parte do instrumento de coleta, referente ao eixo 2 - resultados em evidência foi realizada a análise de conteúdo, caracterizada por uma técnica de investigação que tem por finalidade a descrição objetiva, sistemática e quantitativa do conteúdo manifesto da comunicação (Bardin, 2011). A análise dos artigos foi realizada através da pré-análise, da exploração do material, do tratamento dos resultados obtidos e interpretação. A primeira consistiu em sistematizar os artigos escolhidos e fazer uma leitura superficial para o reconhecimento do material. Na segunda, houve um aprofundamento da leitura já realizada, a fim de identificar o que era mais significativo para este estudo. Já na terceira etapa, os materiais coletados foram discutidos para se tornarem mais convincentes e válidos à pesquisa (Bardin, 2011). 
Assim, com a leitura crítica dos artigos selecionados, os resultados obtidos foram organizados e emergiram as seguintes categorias temáticas: (1) processo de implantação das PICS na atenção básica, (2) aplicação das PICs para variadas finalidades no contexto da atenção básica, (3) formação/capacitação dos profissionais da saúde envolvidos. A discussão dos artigos culminou na revisão integrativa apresentada nos resultados a seguir.

\section{Resultados e Discussão}

Nas buscas realizadas, foram encontrados 148 estudos científicos no total. Após a leitura, privilegiou-se aqueles condizentes com os objetivos do estudo. Esses estudos foram devidamente selecionados pelo fato de seus aspectos apresentarem relação com a proposta do estudo. Dentre os estudos encontrados dentro da temática proposta, 50 se constituíram como amostra final deste levantamento, conforme demonstrado na Figura 1, excluindo-se os de ocorrência repetida e aqueles que não eram artigos completos (relatórios curtos, entrevistas, cartas de editores, prefácios, entre outros).

Figura 1. Fluxograma da descrição da coleta de dados do estudo.

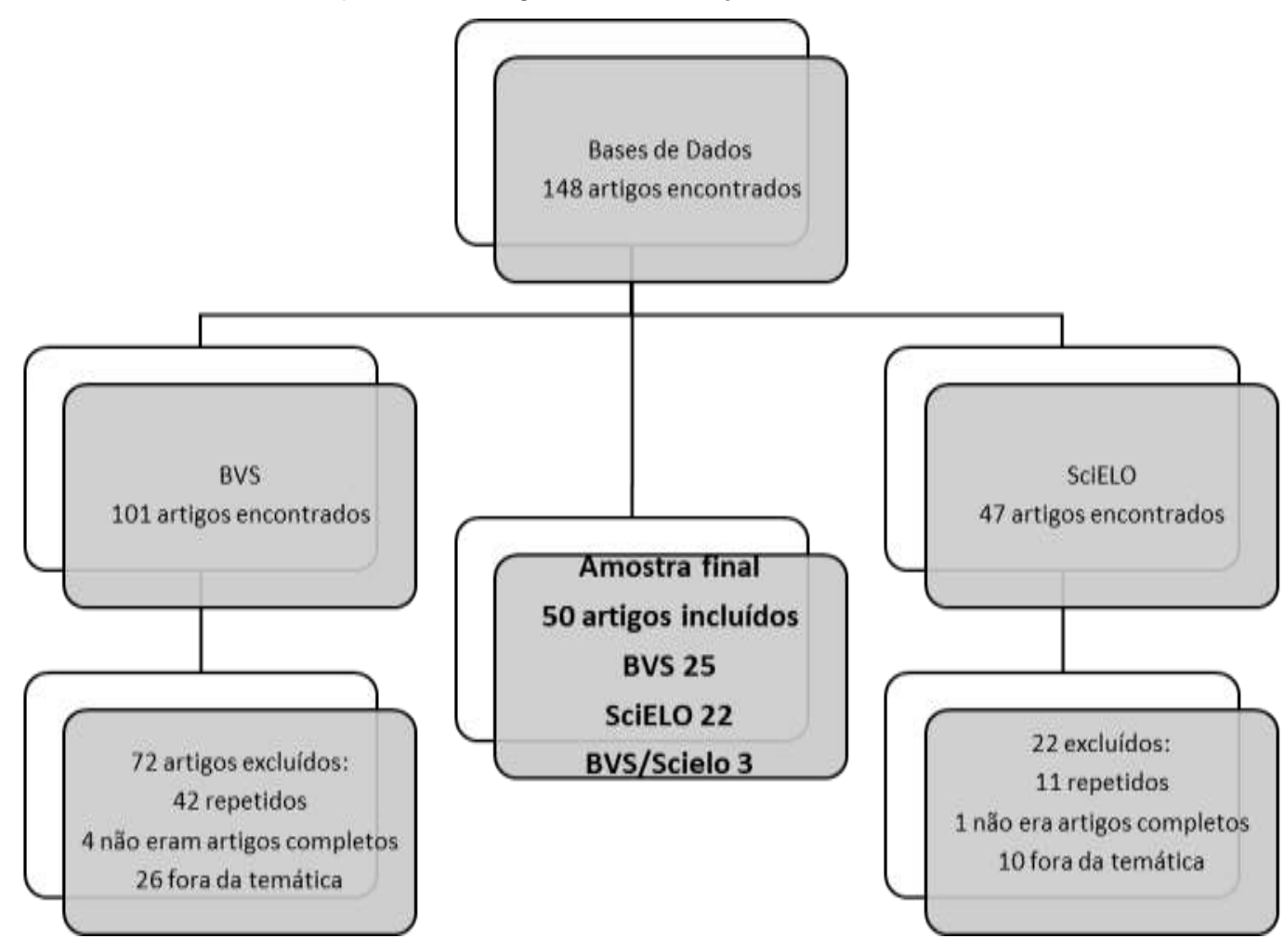

Fonte: Autores.

A caracterização temporal das publicações está apresentada na Tabela 1. No que concerne ao volume de estudos por ano de publicação, evidencia-se que os estudos envolvendo a temática passaram a ser objeto de publicações de forma mais intensa a partir de 2017. O número expressivo de publicações no ano de 2017 coincide com o ano de publicação da Portaria $n^{\circ}$ 849, de 27 de março de 2017 do Ministério da Saúde, que incrementou 14 novas PICs no rol do SUS, trazendo novamente a temática das PICs para o cenário de destaque nacional (Brasil, 2017). 
Tabela 1. Ano de publicação dos artigos incluídos no estudo.

\begin{tabular}{l|ll}
\hline ANO & $\mathbf{N}^{\mathbf{0}}$ DE ARTIGOS & $\%$ \\
\hline $\mathbf{2 0 1 4}$ & 5 & $10 \%$ \\
$\mathbf{2 0 1 5}$ & 8 & $16 \%$ \\
$\mathbf{2 0 1 6}$ & 4 & $8 \%$ \\
$\mathbf{2 0 1 7}$ & 20 & $40 \%$ \\
$\mathbf{2 0 1 8}$ & 13 & $26 \%$ \\
TOTAL & 50 & $100 \%$ \\
\hline
\end{tabular}

Fonte: Autores (2019).

Quanto ao idioma dos artigos selecionados para a amostra, conforme a Tabela 2, há predominância de artigos em língua portuguesa, em virtude dos filtros utilizados e da base de dados Scielo conter apenas publicações brasileiras. Em relação aos artigos em língua estrangeira, há grande número de artigos em inglês, por esta ser a língua predominantemente utilizada no meio científico.

Tabela 2. Idioma dos artigos da amostra.

\begin{tabular}{l|ll}
\hline \multicolumn{1}{l}{ IDIOMA } & $\mathbf{N}^{\mathbf{0}}$ DE ARTIGOS & $\%$ \\
\hline ESPANHOL & 0 & $0 \%$ \\
INGLÊS & 21 & $42 \%$ \\
PORTUGUES & 29 & $58 \%$ \\
TOTAL & 50 & $100 \%$ \\
\hline
\end{tabular}

Fonte: Autores (2019).

Como demonstrado na Tabela 3, quanto à modalidade de pesquisa, houve predominância de pesquisas de campo, caracterizadas por estudos com realização de grupos focais, estudos de caso, aplicação de questionários e entrevistas com usuários de PICs e profissionais da atenção básica. Observou-se também um número significativo de pesquisas bibliográficas (36\%), que são de grande valia para a síntese do conhecimento do objeto de estudo. Além disso, segundo Mendes, Silveira e Galvão (2008), as revisões bibliográficas são muito importantes, porque dão suporte para a tomada de decisão e para a melhoria da prática profissional e apontam lacunas do conhecimento que precisam ser preenchidas com novas pesquisas.

Tabela 3. Modalidade das pesquisas da amostra.

\begin{tabular}{|c|c|c|}
\hline MODALIDADE & $\mathbf{N}^{\circ}$ DE ARTIGOS & $\%$ \\
\hline BIBLIOGRÁFICA & 18 & $36 \%$ \\
\hline DE CAMPO & 30 & $60 \%$ \\
\hline DOCUMENTAL & 2 & $4 \%$ \\
\hline TOTAL & 50 & $100 \%$ \\
\hline
\end{tabular}

Fonte: Autores (2019). 
Assim, para melhor compreensão das categorias temáticas que emergiram, foram assim denominadas: (1) Processo de implantação das PICS na atenção básica, (2) Aplicação das PICs para variadas finalidades no contexto da atenção básica e (3) Formação/capacitação dos profissionais da saúde envolvidos. Nesse contexto, construiu-se quadros ilustrativos que estão apresentados nos apêndices deste trabalho, fazendo referência aos artigos selecionados e contemplados com as seguintes variáveis: título do artigo, periódico, autores, ano de publicação, sujeitos do estudo e tipo de coleta de dados.

Processo de implantação das PICS na atenção básica

Na categoria temática, foram agrupados 15 artigos, os quais reconhecem a importância da implantação das PICS, os benefícios de ter mais um método alternativo de saúde, mas sempre agregado ao modelo biomédico e necessidade de conscientização e sensibilização junto à gestão, população e profissionais especialmente ao nível local.

Sharp et al. (2018) evidenciaram em seu estudo que a implantação dessas terapias no modelo de saúde vigente é limitada por obstáculos estruturais, disputas filosóficas e inquietações sobre a mudança no tipo de cuidado e que torna-se necessário a incorporação de um fluxo e rotinas diferenciado de regulação dos serviços de saúde, de modo a sensibilizar, os profissionais clínicos gerais, os gestores locais sobre a importância da implantação e implementação das PICS na atenção básica.

Para tanto, Brasil (2015) afirma que a implantação e implementação das Práticas Integrativas Complementares são significativas e crescentes no SUS, demandando pensar estrategicamente sua expansão, para o seu fortalecimento para além da atenção básica com o intuito de introduzir um complexo sistema de métodos alternativos, visando o estímulo a mecanismos naturais eficazes e seguros, prezando o processo de humanização no cuidado, através, sobretudo, da construção de um elo entre saúde, profissionais da APS, gestores e meio ambiente, previstos na PNPIC.

Nesse contexto, de acordo os dados do Sistema de Informação da Atenção Básica (SISAB) sobre as PICS no Brasil, as práticas estão implantadas em $78 \%$ dos municípios brasileiros, contemplando 100\% das capitais, sendo que no primeiro semestre de 2017, 4.365 municípios brasileiros ofertavam atendimentos em PICS, principalmente na Atenção básica (Brasil, 2017).

Sousa et al. (2017), defende em sua publicação que a consolidação de práticas diferenciadas no cuidado, antecede um complexo de políticas emancipatórias que pressupõe maior participação das pessoas no cuidado e consolida um sistema realmente pautado na universalidade e integralidade. Como afirma o estudo de Paranaguá et al (2009) que a forma de assistência defendida junto a utilização das práticas integrativas no SUS contemplam de fato aos princípios doutrinários do SUS: - da universalidade do cuidado mais humano e de modo integral em saúde, além da qualidade, eficácia e segurança no cuidado prestado.

Aplicação das PICS para variadas finalidades no contexto da atenção básica

Agrupou-se 26 artigos nessa categoria temática, onde são abordados diversos subtemas a respeitos dos tipos de PICs que podem ser utilizadas em condições de saúde, por exemplo: saúde mental, condições neurológicas crônicas, alergias, gravidez e distúrbios cardiovasculares. 
Em relação à saúde mental, Shah et al. (2017) resumem as evidências clínicas de cinco principais abordagens de tratamento integrativo para o manejo de humor: espiritualidade, ervas e suplementos, nutrição, terapias mente-corpo e atividades de lazer. No que tange às condições neurológicas crônicas, neuropatia diabética, enxaqueca e demência são as condições onde as intervenções da medicina integrativa e complementar são mais frequentemente utilizadas pelos profissionais da atenção básica. A maioria dos pacientes faz uso de intervenções mente-corpo, tais como exercícios de respiração, meditação e yoga, porém, são necessários mais estudos para apoiar as evidências preliminares dos benefícios nessas condições (Bega,2017).

Várias terapias demonstraram resultados promissores em estudos randomizados, tais como: acupuntura e sprays nasais com derivados de ervas (capsaicina e celulose inerte) para tratamento de rinite alérgica; e tratamento para asma com ervas da Medicina Tradicional Chinesa, como Mai Men Dong Tang (Quiu; Grine, 2016).

Sobre o uso de PICs na gravidez de baixo risco, a literatura revela que há evidências fortes de que terapias mente-corpo como yoga e meditação mindfulness proporcionam às mulheres trabalhos mais curtos, menos partos assistidos por instrumentos e redução do estresse e ansiedade, além de bebês com maior peso ao nascer. Além disso, a acupuntura se mostrou um método eficaz e sem contraindicações para a indução do trabalho de parto (Cuneo, 2017).

No que concerne aos distúrbios cardiovasculares, Mehta (2017) destaca a necessidade da realização de mais estudos sobre a aplicação de PICs, tendo em vista evidências preliminares de benefícios da yoga, tai-chi e acupuntura na melhoria da qualidade de vida de pacientes pós acidente vascular encefálico (AVE) e ataque cardíaco; de musicoterapia e terapias manuais, como quiropraxia e massagens, no pós-operatório de doenças valvares; e da yoga na melhoria de arritmias cardíacas.

Dessa forma, Chung et al. (2016) afirmam que a camada da população com menor acesso aos tratamentos de saúde convencionais e menor proteção social tentem a fazer uso da medicina complementar como único recurso disponível. Diante desse quadro, é importante frisar o papel das PICs como agentes complementares e não alternativos ao tratamento convencional e a importância de deixar isso bem claro para os pacientes.

Formação/capacitação dos profissionais da saúde envolvidos

A categoria temática emergida aponta como desafios, os seguintes eixos comuns apresentados: a dificuldade na formação e qualificação de profissionais em número adequado para atuarem no SUS, enfatizando que a formação em saúde, de modo geral, ainda precisa avançar no sentido de preparar profissionais no atendimento aos pressupostos do SUS, bem como uma escassa abordagem a inserção da disciplina PICS na matriz curricular dos cursos de graduação e pós graduação e formação complementar.

O estudo de Lopes et al (2018), evidenciou que há interesse por parte dos acadêmicos e profissionais da saúde sobre o tema, no entanto, um fator considerado como dificultador é o fato de não ser ofertada disciplinas nos cursos da saúde. Nesse sentido, Reis, Esteves e Greco (2018) afirmam em seu estudo que há um escasso enfoque em informações sobre as PICs no espaço acadêmico, e isso pode ser atribuído na maioria das vezes as limitações pertinentes à pouca valorização de uma visão holística no cuidado em saúde, má gestão no sistema público e compreensões enraizadas na medicina alopática, demonstrando assim a necessidade de mais investimentos em educação permanente e na matriz curricular para os profissionais que atuam nos serviços públicos de saúde. 
Dessa forma, de acordo com Teixeira e Lin (2013), acredita-se que de maneira alternativa, complementar ou agregada ao modelo biomédico convencional, a utilização das PICs vem sendo uma realidade nos serviços de saúde e empregadas em todos os países e por todas as classes sociais da população. Outrossim, esse cenário demanda ao profissional médico, compreensão dessas terapias, com intuito de que possa orientar os usuários dos serviços de saúde que que desejem utilizar tratamentos diferentes dos que está acostumado a prescrever.

De modo mais abrangente fazendo referência a categoria temática formação/treinamento das PICs emergida dos artigos científicos outrora apresentados, apresenta-se um panorama necessário de implementação da matriz curricular dos cursos de graduação e pós graduação, bem como de educação permanente com vistas a contemplar as PICs, com intuito de fortalecer o processo saúde-doença e cuidado.

\section{Conclusão}

Diante de todo o exposto, pode-se concluir que as PICs estão cada vez mais em voga no cenário científico nacional e mundial. Em virtude do seu potencial integrativo e relativo baixo custo de implantação, essas técnicas representam um arsenal terapêutico de grande valia para aplicação na atenção básica como complemento aos tratamentos convencionais.

Embora a busca por esses serviços esteja cada vez maior, a produção científica sobre a temática ainda necessita de muitos avanços, sobretudo na validação científica e na proposição de métodos que comprovem os benefícios das PICs em diversos contextos de saúde. Além disso, a formação de profissionais capacitados ainda necessita ser bastante ampliada, com inclusão nas matrizes curriculares dos cursos de medicina e demais cursos da área da saúde, promoção de debates nas universidades, e combate ao preconceito e à visão puramente biológica e mecanicista do corpo humano. A partir disso e da continuidade das políticas públicas de incentivo ao uso das PICs no SUS, poderão ser vencidos os desafios apresentados para a implantação efetiva da PNPIC, ampliando cada vez mais o acesso da população a um atendimento de saúde integral.

\section{Referências}

Bardin L. (2011). Análise de Conteúdo. Edições 70.

Bega D (2017). Complementary and integrative interventions for chronic neurologic conditions encountered in the primary care office. Prim Care Clin Office Pract, 44, 305-322.

Brant L.C. et al (2014). Práticas integrativas e complementares: os desafios da implantação de uma política. Revista Eletrônica Gestão \& Saúde, 5, 843-861.

Brasil. (2015). Política nacional de práticas integrativas e complementares no SUS: atitude de ampliação de acesso / Ministério da Saúde. Secretaria de Atenção à Saúde. Departamento de Atenção Básica. (2a ed.), Ministério da Saúde.

Brasil (2011). Portaria nº 2.488, de 21 de outubro de 2011. Aprova a Política Nacional de Atenção Básica, estabelecendo a revisão de diretrizes e normas para a organização da Atenção Básica, para a Estratégia Saúde da Família (ESF) e o Programa de Agentes Comunitários de Saúde (PACS). Diário Oficial da União, Brasília, DF.

Brasil (2017). Portaria n 849, de 27 de março de 2017. Inclui a Arteterapia, Ayurveda, Biodança, Dança Circular, Meditação, Musicoterapia, Naturopatia, Osteopatia, Quiropraxia, Reflexoterapia, Reiki, Shantala, Terapia Comunitária Integrativa e Yoga à Política Nacional de Práticas Integrativas e Complementares. Diário Oficial da União, Brasília, DF.

Brasil (2018). Portaria ${ }^{\circ}$ 702, de 21 de março de 2018. Altera a Portaria de Consolidação n $2 / G M / M S$, de 28 de setembro de 2017 , para incluir novas práticas na Política Nacional de Práticas Integrativas e Complementares - PNPIC. Diário Oficial da União, Brasília, DF.

Chung, V. C. H. et al (2016). Use of Traditional and Complementary Medicine as Self-Care Strategies in Community Health Centers Cross-Sectional Study in Urban Pearl River Delta Region of China. Medicine, 95, 23.

Cuneo J (2017). Women's Health Pregnancy and Conception. Prim Care Clin Office Pract, 44, 369-376.

Habimorad P. H. L (2015). Práticas integrativas e complementares no SUS: revisão integrativa [dissertação]. Botucatu: Universidade Estadual Paulista "Júlio de Mesquita Filho", Faculdade de Medicina de Botucatu.

Losso L. N., \& Lopes S. S. (2017). Análise da oferta e produção de atendimento em acupuntura na atenção básica em Santa Catarina. Tempus Actas de Saúde Coletiva, 11, 159-177. 
Research, Society and Development, v. 10, n. 9, e42910918204, 2021

(CC BY 4.0) | ISSN 2525-3409 | DOI: http://dx.doi.org/10.33448/rsd-v10i9.18204

Mehta D (2017). Integrative Medicine and Cardiovascular Disorders. Prim Care Clin Office Pract, 44, 351-367.

Mendes k. D. S., Silveira R. C. C. P., \& Galvao C. M (2008). Revisão integrativa: método de pesquisa para a incorporação de evidências na saúde e na enfermagem. Texto contexto - enferm. 17, 758-764.

Ministério da saúde. Departamento de Atenção básica (2018). Política Nacional de Práticas Integrativas e Complementares no SUS, s.d.

Qiu J, \& Grine K (2016) Complementary and Alternative Treatment for Allergic Conditions. Prim Care Clin Office Pract, 43, 519-526.

Reis B. O., Esteves L. R, \& Greco R. M. (2018) Avanços e desafios para a implementação das práticas integrativas e complementares no brasil. Revista de Atenção Primaria a Saúde, 21(3).

Rodrigues A.J (2006). Metodologia científica. Avercamp.

Shah A. K., Becicka R. T. M. R., Edberg D., \& Namboodiri S (2017). Integrative Medicine and Mood, Emotions and Mental Health. Prim Care Clin Office Pract, 44, 281-304.

Sharp D. et al (2018). 'Trying to put a square peg into a round hole': a qualitative study of healthcare professionals' views of integrating complementary medicine into primary care for musculoskeletal and mental health comorbidity. BMC Complement Altern Med., 18, $\mathrm{n}$

Sousa L. A. et al (2017). Acupuntura no Sistema Único de Saúde - uma análise nos diferentes instrumentos de gestão. Ciênc. saúde coletiva, 22, 301-310.

Souza M. T., Silva M. D, \& Carvalho R (2010). Revisão integrativa: o que é e como fazer. Einstein. 8, 102-106.

Teixeira M. Z., \& Lin Chin Na (2013). Educação médica em terapêuticas não convencionais. Revista de Medicina, 92, 224-235.

Ursi E. S (2005). Prevenção de lesões de pele no perioperatório: revisão integrativa da literatura [dissertação]. Universidade de São Paulo, Escola de Enfermagem de Ribeirão Preto. 